\title{
Quantum Blahut-Arimoto Algorithms
}

\author{
Navneeth Ramakrishnan*§, Raban Iten ${ }^{\dagger \S}$, Volkher Scholz ${ }^{\ddagger}$ and Mario Berta* \\ *Department of Computing, Imperial College London, United Kingdom \\ ${ }^{\dagger}$ Institute for Theoretical Physics, ETH Zurich, Switzerland \\ $\ddagger$ Department of Physics and Astronomy, Ghent University, Belgium
}

\begin{abstract}
We generalize alternating optimization algorithms of Blahut-Arimoto type to the quantum setting. In particular, we give iterative algorithms to compute the mutual information of quantum channels, the thermodynamic capacity of quantum channels, the coherent information of less noisy quantum channels, and the Holevo quantity of classical-quantum channels. Our convergence analysis is based on quantum entropy inequalities and leads to $a$ priori additive $\varepsilon$-approximations after $\mathcal{O}\left(\varepsilon^{-1} \log N\right)$ iterations, where $N$ denotes the input dimension of the channel. We complement our analysis with an a posteriori stopping criterion which allows us to terminate the algorithm after significantly fewer iterations compared to the $a$ priori criterion in numerical examples. Finally, we discuss heuristics to accelerate the convergence.
\end{abstract}

\section{INTRODUCTION}

In quantum information theory, many quantities of interest are given by convex optimization problems that can often not be solved analytically. Consequently, numerical tools that provably find approximate solutions in an efficient manner are sought after. In our work, we are primarily interested in the efficient calculation of entropic channel capacity formulas from quantum Shannon theory while giving rigorous guarantees on the approximation error dependent on the number of steps in the algorithm. For example, by the Holevo-SchumacherWestmoreland theorem the classical capacity of classicalquantum (cq) channels is given by the Holevo quantity [1], [2]. This then represents a convex optimization problem over the set of input probability distributions. Various methods to approximate the solution of such convex optimization problems in quantum information theory have been proposed in the literature [3], [4], [5], [6], [7], [8], [9], [10], [11], [12], [13].

In this work, we study alternating optimization algorithms and give quantum extensions of the Blahut-Arimoto algorithm from classical information theory [14], [15]. Blahut-Arimoto type algorithms are specifically tailored for entropic problems and have analytic convergence guarantees derived from entropy inequalities (see [16], [17], [18], [19], [20], [21], [22] for classical extensions of the original works). Accelerated Blahut-Arimoto algorithms with faster analytical and numerical convergence are also known [23], [24], [25], [26]. While there have been works on Blahut-Arimoto inspired algorithms to calculate the capacity of classical-quantum channels [3], [27], this work generalizes the Blahut-Arimoto algorithm to the fully quantum setting.

\footnotetext{
$\S$ These authors contributed equally
}

\section{OVERVIEW OF RESULTS}

We present algorithms to compute the mutual information of quantum channels, the thermodynamic capacity of quantum channels, the coherent information of less noisy quantum channels, and the Holevo quantity of classical-quantum channels. As special cases of the thermodynamic capacity, the minimal entropy gain and the completely bounded minimal conditional entropy are covered as well. The algorithms and the convergence proofs are based on the quantum relative entropy which is defined for positive semi-definite operators ${ }^{1}$ $\rho$ and $\sigma$ as

$$
D(\rho \| \sigma)= \begin{cases}\operatorname{Tr}[\rho(\log \rho-\log \sigma)] & \text { if } \sigma \gg \rho \\ \infty & \text { otherwise }\end{cases}
$$

where the notation $\sigma \gg \rho$ denotes that the kernel of $\sigma$ is a subset of the kernel of $\rho$, and where we take the logarithm only on the support of the argument. In particular, we use the fact that the relative entropy satisfies certain inequalities under the action of channels such as the data processing inequality, which states that for all $\rho, \sigma$ we have $D(\mathcal{E}(\rho) \| \mathcal{E}(\sigma)) \leq D(\rho \| \sigma)$.

The number of iterations required by our algorithm to obtain an a priori error of $\varepsilon$ is $\mathcal{O}\left(\varepsilon^{-1} \log N\right)$, where $N$ denotes the input dimension. Moreover, one can construct an accelerated version of the algorithm similar to the classical case [24], [25] to lower the number of iterations required by a constant factor compared to the standard version. This procedure also gives rise to heuristics that provide a significant speed-up of the algorithm in our numerics.

Other algorithms for the classical-quantum case include that of Sutter et al. [9] based on convex programming duality and smoothing techniques. This algorithm has a time complexity of $\mathcal{O}\left(\varepsilon^{-1} \max \{|X|,|B|\}|B|^{3} \sqrt{\log |X|}\right)$ versus our $\mathcal{O}\left(\varepsilon^{-1}\left(|B|^{3}+|B|^{2}|X|\right) \log |X|+|X||B|^{3}\right)$. This suggests that our iterative algorithm is faster for the regime $|B| \gg|X|$ but slower for $|X| \gg|B|$ in the worst case. For other algorithms that compute the Holevo quantity, there is often no explicit complexity analysis given. Comparing with the relative entropy optimization work of Fawzi and Fawzi [10] — based on semi-definite approximations of the matrix logarithm [11] — our numerics suggest that for the specific optimization problems considered here, Blahut-Arimoto

\footnotetext{
${ }^{1}$ Here and henceforth, any quantum definition applies to classical probability distributions as well, by embedding them as matrices diagonal in some prefixed basis.
} 
type algorithms converge faster. We emphasize that while the advantage of Blahut-Arimoto type algorithms might be explained by their conformity to the specific structure of entropy optimization problems, the method presented in [10] is applicable for a wider range of problems.

The a priori bound on the error corresponds to the worst case and the number of iterations required is extremely conservative for most problems. Hence, we adapt classical techniques [17] to find a posteriori error bounds on the capacity estimate at each iteration of the algorithm. This error bound can then be used as a termination criterion for our numerics. We notice that for the channels and capacities we consider, the a posteriori error is achieved in significantly fewer iterations compared to the a priori requirement. One of the explanations for this speed-up is that the convergence of our algorithm can be exponentially fast in certain cases. Specifically, when the quantum relative entropy inequalities used by our convergence proof are strict inequalities, one can show (see Proposition 3.5 for more details) that the error in the capacity estimate after at the $t^{\text {th }}$ iteration for a channel with input dimension $N$ is bounded by $(1-\delta)^{t} \log N$ for some $\delta>0$.

\section{Structure OF QUANTUM Blahut-ARIMoto ALGORITHMS}

\section{A. Notation}

We label Hilbert spaces (which are assumed to be finitedimensional) with capital letters e.g. $A, B$, and denote their dimension by $|A|,|B|$, and so on. The set of density operators on a system $A$, i.e., positive semi-definite matrices $\rho_{A}$ with $\operatorname{Tr}\left[\rho_{A}\right]=1$, is denoted $\mathcal{D}(A)$. Whenever we work with a single system, we may omit the sub-index referring to the system. A quantum channel $\mathcal{E}_{A \rightarrow B}$ is a linear completely positive trace-preserving map that maps density operators on a system $A$ to density operators on a system $B$. The complementary channel $\mathcal{E}_{A \rightarrow E}^{c}$ of a channel $\mathcal{E}_{A \rightarrow B}$ is defined via taking the partial trace over $B$ of the Stinespring dilation $U_{A \rightarrow B E}$ of $\mathcal{E}_{A \rightarrow B}$. For simplicity, we may write $\mathcal{E}$ and $\mathcal{E}_{c}$ instead of $\mathcal{E}_{A \rightarrow B}$ and $\mathcal{E}_{A \rightarrow E}^{c}$ if the input and output systems of the channel are clear from the context.

For a density operator $\rho$, the von Neumann entropy is defined as $S(\rho)=-\operatorname{Tr}[\rho \log \rho]$ and for density operators $\rho, \sigma$ the quantum relative entropy is defined as in (1). We also work with discrete probability distributions, which we represent as vectors $\lambda=\left[\lambda_{1}, \ldots, \lambda_{m}\right]$ with $\sum_{i} \lambda_{i}=1$ or alternatively as diagonal matrices with entries $\lambda_{1}, \ldots, \lambda_{m}$ i.e., $\rho_{\lambda}=\sum_{i=1}^{m} \lambda_{i}|i\rangle\langle i|$ in some pre-defined basis.

\section{B. Blahut-Arimoto type}

Proofs for this section are available in the full version of our work [28]. We now study a special entropic type of alternating optimization algorithms, called Blahut-Arimoto algorithms [14], [15]. The channel capacities we are interested in are typically given as a convex optimization problem over input states. One may write an extension function $J$ in two variables such that the maximization over both variables gives back the capacity of the channel. Performing the maximizations iteratively leads to an algorithm of the following form.

Algorithm 1 Blahut-Arimoto algorithm: Iterative double optimization over density operators

1: Inputs:

- Initial guess $\rho_{A}^{(1)} \in \mathcal{D}(A)$ with full support, i.e., $\rho_{A}^{(1)}>$ 0

- Function $J_{\gamma}: \mathcal{D}(A) \times \mathcal{D}(B) \mapsto \mathbb{R}$ with a coefficient $\gamma>0$

- Update relations $\mathcal{F}_{1}: \mathcal{D}(A) \mapsto \mathcal{D}(B)$ and $\mathcal{F}_{2}$ : $\mathcal{D}(B) \mapsto \mathcal{D}(A)$

- Number of iteration steps $n$

2: for $t \in\{1,2, \ldots, n\}$ do

3: $\quad \sigma_{B}^{(t)}=\mathcal{F}_{1}\left(\rho_{A}^{(t)}\right)$

4: $\quad \rho_{A}^{(t+1)}=\mathcal{F}_{2}\left(\sigma_{B}^{(t)}\right)$

5: end for

6: Outputs: $\rho_{A}^{(n+1)}, C(n)=J_{\gamma}\left(\rho_{A}^{(n+1)}, \sigma_{B}^{(n)}\right)$, where $C(n)$ should approximate $C^{\star}=\max _{\rho_{A}, \sigma_{B}} J_{\gamma}\left(\rho_{A}, \sigma_{B}\right)$ for $n \rightarrow$ $\infty$.

Definition 3.1 (Blahut-Arimoto): Algorithm 1 together with the following conditions on $J, \mathcal{F}_{1}$ and $\mathcal{F}_{2}$ defines a quantum Blahut-Arimoto algorithm: For $\gamma>0$ and density operators $\sigma \gg \rho$,

$$
J_{\gamma}(\rho, \sigma)=-\gamma D(\rho \| \sigma)+\operatorname{Tr}[\rho \mathcal{F}(\sigma)] \in \mathbb{R},
$$

where $\mathcal{F}$ is a Hermitian matrix valued super-operator on density operators such that $\operatorname{Tr}[\rho \mathcal{F}(\sigma)]$ is continuous in $\sigma$ for $\sigma \gg \rho$. The update rules are then given by

$$
\mathcal{F}_{1}(\rho)=\underset{\sigma \text { with } \sigma \gg \rho}{\arg \max } J_{\gamma}(\rho, \sigma), \quad \mathcal{F}_{2}(\sigma)=\underset{\rho \text { with } \sigma \gg \rho}{\arg \max } J_{\gamma}(\rho, \sigma) .
$$

Now, under certain conditions we can find analytic expressions for the optimizers in (3).

Lemma 3.2 (Update rules): For quantum Blahut-Arimoto algorithms with,

$$
\operatorname{Tr}[\rho\{\mathcal{F}(\sigma)-\mathcal{F}(\rho)\}] \leq \gamma D(\rho \| \sigma)
$$

for all density operators $\sigma \gg \rho$, the update rules appearing in Algorithm 1 are of the form

$$
\begin{aligned}
& \mathcal{F}_{1}(\rho)=\rho, \\
& \mathcal{F}_{2}(\sigma)=\frac{1}{Z(\sigma)} \exp \left(\log \sigma+\frac{1}{\gamma} \mathcal{F}(\sigma)\right),
\end{aligned}
$$

with $Z(\sigma)=\operatorname{Tr}\left[\exp \left(\log \sigma+\frac{1}{\gamma} \mathcal{F}(\sigma)\right)\right]$ for density operators $\rho$ and $\sigma>0$.

The next theorem shows convergence of quantum BlahutArimoto algorithms under some technical assumptions.

Theorem 3.3 (Convergence): For quantum Blahut-Arimoto algorithms together with a strictly positive definite initial state $\rho^{(1)}>0$ on a system $A$ and

$$
0 \leq \operatorname{Tr}[\rho\{\mathcal{F}(\sigma)-\mathcal{F}(\rho)\}] \leq \gamma D(\rho \| \sigma),
$$


for density operators $\sigma \gg \rho$, we have that $C(n)$ of Algorithm 1 is monotonically increasing and converges for $n \rightarrow \infty$ to

$$
C^{\star}=\max _{\rho, \sigma \text { with } \sigma \gg \rho} J_{\gamma}(\rho, \sigma)
$$

with the approximation error bounded as

$$
\left|C^{\star}-C(n)\right| \leq \frac{\gamma D\left(\rho^{\star} \| \rho^{(1)}\right)}{n},
$$

where $\rho^{\star}$ is the optimizer (possibly not unique) that achieves the capacity $C^{\star}$. If $\rho^{(1)}$ is chosen to be the maximally mixed state, the approximation error is bounded as $\left|C^{\star}-C(n)\right| \leq$ $\frac{\gamma \log |A|}{n}$.

The value of the positive parameter $\gamma$ appearing in Definition 3.1 should be chosen as small as possible while still respecting the second inequality in (7). For all the quantities we consider, this inequality is a relative entropy inequality under the action of the channel such as the data processing inequality. There exists a value of $\gamma$ for which this holds for all channels and this corresponds to the standard BlahutArimoto algorithm. For certain channels, the inequality may be strict and in these cases, a smaller value of $\gamma$ may be chosen without violating (7). This corresponds to the accelerated Blahut-Arimoto algorithm where the acceleration parameter $\gamma$ is

$$
\gamma=\sup \frac{\operatorname{Tr}[\rho\{\mathcal{F}(\sigma)-\mathcal{F}(\rho)\}]}{D(\rho \| \sigma)},
$$

with the supremum taken over $\sigma \gg \rho$ and $\rho \neq \sigma$. If the right hand side of (10) cannot be efficiently computed, we may also use the following heuristic similar to the classical case [24]. Remark 3.4 (Acceleration heuristic): For $D\left(\rho^{(t)} \| \rho^{(t-1)}\right) \neq 0$, define an adaptive acceleration parameter

$$
\gamma^{(t+1)}=\frac{\operatorname{Tr}\left[\rho^{(t)}\left\{\mathcal{F}\left(\rho^{(t-1)}\right)-\mathcal{F}\left(\rho^{(t)}\right)\right\}\right]}{D\left(\rho^{(t)} \| \rho^{(t-1)}\right)} .
$$

The proof of convergence from Theorem 3.3 no longer holds under the adaptive acceleration heuristic. However, we find in practice that it provides a significant speed-up as shown in Figure 1.

Strictness in the second inequality of (7) led to an accelerated algorithm. Similarly, for some channels, the first inequality of (7) may be strict. When this is the case, the speed of convergence is exponential.

Proposition 3.5 (Exponential convergence): For quantum Blahut-Arimoto algorithms satisfying

$$
0<\inf \operatorname{Tr}[\rho\{\mathcal{F}(\sigma)-\mathcal{F}(\rho)\}],
$$

for all density operators $\sigma \gg \rho$ and $\sigma \neq \rho$, there exists $\delta>0$ such that after $t$ iterations

$$
\left|C^{\star}-C(t)\right| \leq(1-\delta)^{t-1} \gamma D\left(\rho^{\star}|| \rho^{(1)}\right) .
$$

Moreover, when $\rho^{(1)}$ is chosen to be the maximally mixed state, we obtain $\left|C^{\star}-C(t)\right| \leq(1-\delta)^{t-1} \gamma \log |A|$. Details on how the value of $\delta$ depends on the strictness of (12) can be found in [28].
Finally, the following proposition allows us to terminate the algorithm when the a posteriori error is sufficiently small. Proposition 3.6 (Termination criteria): For quantum BlahutArimoto algorithms that satisfy (7) let us denote the largest eigenvalue of $\mathcal{F}\left(\rho^{(t)}\right)$ by $\alpha_{\max }^{(t)}$ for $t \in \mathbb{N}$. Then, we have

$$
C(t) \leq C^{\star} \leq \alpha_{\max }^{(t)}
$$

Hence, in particular, $\left|C^{\star}-C(t)\right| \leq \alpha_{\max }^{(t)}-C(t)$ provides a bound on the a posteriori error at every iteration.

In particular, if $\rho^{(t)}$ converges to a full rank optimizer $\rho^{\star}$, then we may take the logarithm on both sides of (6) to show that $\lim _{t \rightarrow \infty} \alpha_{\max }^{(t)}=C^{\star}$. This shows that the a posteriori criterion is indeed achieved for some sufficiently large $t$ for full rank optimizers.

\section{EXAMPLE - COHERENT INFORMATION OF LESS NOISY CHANNELS}

For a quantum channel $\mathcal{E}_{A \rightarrow B}$ with complementary channel $\left(\mathcal{E}_{c}\right)_{A \rightarrow E}$, the coherent information of the channel, $I_{\text {coh }}(\mathcal{E})$, is defined as the maximum of the coherent information $I_{\text {coh }}(\rho, \mathcal{E})=S(\mathcal{E}(\rho))-S\left(\mathcal{E}_{c}(\rho)\right)$, where the maximization is taken over input states $\rho$. That is, we have

$$
I_{\mathrm{coh}}(\mathcal{E})=\max _{\rho} \underbrace{S(\mathcal{E}(\rho))-S\left(\mathcal{E}_{c}(\rho)\right)}_{=I_{\mathrm{coh}}(\rho, \mathcal{E})} .
$$

A channel $\mathcal{E}$ is called less noisy if the private capacity of its complementary channel $\mathcal{E}_{c}$ is zero [29]. This implies that for all density operators $\rho$ and $\sigma$, we have

$$
D(\mathcal{E}(\rho) \| \mathcal{E}(\sigma)) \geq D\left(\mathcal{E}_{c}(\rho) \| \mathcal{E}_{c}(\sigma)\right) .
$$

The quantum capacity $Q(\mathcal{E})$ of the channel $\mathcal{E}$ is the regularized coherent information of a channel [30] and corresponds to the rate at which Alice can transmit quantum information to Bob. In [29] it is shown that the coherent information of a channel is additive if the channel is less noisy. Hence, for less noisy channels, we have $Q(\mathcal{E})=I_{\text {coh }}(\mathcal{E})$. We shall only consider less noisy channels in this section and thus drop the distinction between the regularized and single letter formulas.

\section{A. Blahut-Arimoto algorithm}

The coherent information of a less noisy channel can be estimated using a Blahut-Arimoto algorithm. We define the following two variable extension of $I_{\operatorname{coh}}(\rho, \mathcal{E})$ for $\sigma \gg \rho$.

$$
\begin{aligned}
J_{\gamma}(\rho, \sigma, \mathcal{E}) & =I_{\mathrm{coh}}(\rho, \mathcal{E})+D(\mathcal{E}(\rho) \| \mathcal{E}(\sigma)) \\
& -D\left(\mathcal{E}_{c}(\rho) \| \mathcal{E}_{c}(\sigma)\right)-\gamma D(\rho \| \sigma) .
\end{aligned}
$$

With a short calculation, one can bring $J_{\gamma}$ into the following form

$$
J_{\gamma}(\rho, \sigma, \mathcal{E})=-\gamma \operatorname{Tr}[\rho \log \rho]+\operatorname{Tr}[\rho\{\gamma \log \sigma+\mathcal{F}(\sigma)\}]
$$

where $\mathcal{F}(\sigma)=\mathcal{E}_{c}^{\dagger} \log \mathcal{E}_{c}(\sigma)-\mathcal{E}^{\dagger} \log \mathcal{E}(\sigma)$. Substituting $\mathcal{F}$ into (10), we obtain $\gamma \geq \zeta_{\text {con }}$, where

$$
\zeta_{\text {con }}(\mathcal{E})=\sup \frac{D(\mathcal{E}(\rho) \| \mathcal{E}(\sigma))-D\left(\mathcal{E}_{c}(\rho) \| \mathcal{E}_{c}(\sigma)\right)}{D(\rho \| \sigma)},
$$


for $\rho \neq \sigma$ and $\sigma \gg \rho$. Note that by the non-negativity of relative entropy and the data processing inequality, we have $\zeta_{\text {con }}(\mathcal{E}) \in[-1,1]$ for all $\mathcal{E}$ but for less noisy channels, (16) implies that $\zeta_{\text {con }}(\mathcal{E}) \in[0,1]$. Indeed, $\gamma=1$ corresponds to the standard algorithm and if $\zeta_{\text {con }}(\mathcal{E})<1$, then one may accelerate the algorithm. Moreover, another short calculation leads to

$$
\operatorname{Tr}[\rho\{\mathcal{F}(\sigma)-\mathcal{F}(\rho)\}]=D(\mathcal{E}(\rho) \| \mathcal{E}(\sigma))-D\left(\mathcal{E}_{c}(\rho) \| \mathcal{E}_{c}(\sigma)\right)
$$$$
\Longrightarrow 0 \leq \operatorname{Tr}[\rho\{\mathcal{F}(\sigma)-\mathcal{F}(\rho)\}] \leq \gamma D(\rho \| \sigma),
$$

where we have used (16) along with $\gamma \geq \zeta_{\text {con }}(\mathcal{E})$. From Lemma 3.2, whose requirement is satisfied by (21), we find a double optimization form of the coherent information $I_{\mathrm{coh}}(\mathcal{E})$

$$
\max _{\rho, \sigma \text { with } \sigma \gg \rho} J_{\gamma}(\rho, \sigma, \mathcal{E})=I_{\text {coh }}(\mathcal{E}) .
$$

Performing the two maximizations in $\max _{\rho, \sigma} J_{\gamma}(\rho, \sigma, \mathcal{E})$ iteratively, leads to Algorithm 2 (see Lemma 3.2 for the form of the update rules), which provably converges to the coherent information by Theorem 3.3.

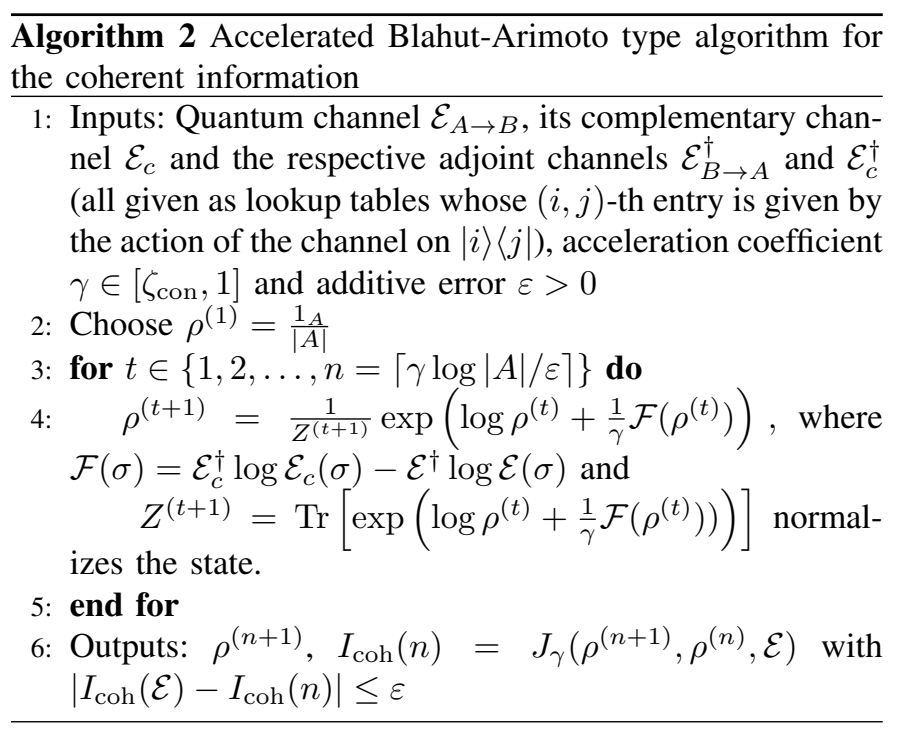

We also have exponential convergence for strictly less noisy channels with

$$
\inf D(\mathcal{E}(\rho) \| \mathcal{E}(\sigma))-D\left(\mathcal{E}_{c}(\rho) \| \mathcal{E}_{c}(\sigma)\right)>0
$$

where the infimum is over $\rho \neq \sigma$ and $\sigma \gg \rho$. To see this, we define

$$
\zeta_{\exp }(\mathcal{E})=\inf \frac{D(\mathcal{E}(\rho) \| \mathcal{E}(\sigma))-D\left(\mathcal{E}_{c}(\rho) \| \mathcal{E}_{c}(\sigma)\right)}{D(\rho \| \sigma)} .
$$

In general, $\zeta_{\exp }(\mathcal{E}) \in[-1,1]$ but for less noisy channels, we have that $\zeta_{\exp }(\mathcal{E}) \in[0,1]$ due to $(16)$. If $\zeta_{\exp }(\mathcal{E})>0$, we get exponential convergence according to Proposition 3.5. Using (20), we see that the condition $\zeta_{\exp }(\mathcal{E})>0$ follows for strictly less noisy channels.

\section{B. Numerics}

We consider the qubit amplitude damping channel whose form is given as

$$
\mathcal{E}_{p}^{A D}(\rho)=A_{0} \rho A_{0}^{\dagger}+A_{1} \rho A_{1}^{\dagger},
$$

with $A_{0}=|0\rangle\langle 0|+\sqrt{1-p}| 1\rangle\left\langle 1\left|, A_{1}=\sqrt{p}\right| 0\right\rangle\langle 1|$ for $p \in$ $[0,1]$. We choose an additive error threshold of $\varepsilon=10^{-6}$ and use the termination criterion given in Proposition 3.6, which significantly reduces the number of iterations of the algorithm required to achieve an estimate of the capacity with additive error smaller than $\varepsilon$. The results are shown in Figure 1. The standard algorithm and the adaptive accelerated algorithm converge in 24 and 5 iterations, respectively.

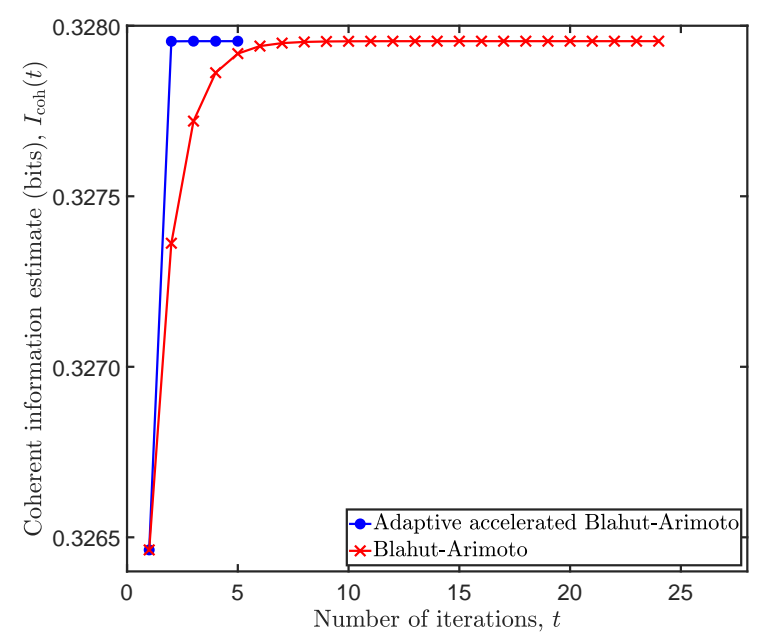

Fig. 1. Convergence of the Blahut-Arimoto algorithm to the coherent information of the amplitude damping channel $\mathcal{E}_{0.3}^{A D}$ given in $(25)$ in the standard and adaptive accelerated case with acceleration parameter $\gamma^{(t)}$ in the $t$-th iteration step as defined in (11). The figure shows the lower bound on the coherent information in each iteration step $t$ until the a posteriori bound given in Proposition 3.6 ensures that we terminate when $\left|C^{\star}-C(t)\right| \leq 10^{-6}$. The adaptive accelerated Blahut-Arimoto algorithm converges after 5 iterations, while the standard algorithm converges after 24 iterations.

\section{Time complexity}

The time complexity of Algorithm 2 is determined by the required number of iterations and the complexity of applying the following update rule

$$
\rho^{(t+1)}=\frac{1}{Z^{(t+1)}} \exp \left(\log \rho^{(t)}+\frac{1}{\gamma} \mathcal{F}\left(\rho^{(t)}\right)\right),
$$

where $\mathcal{F}(\sigma)=\mathcal{E}_{c}^{\dagger} \log \mathcal{E}_{c}(\sigma)-\mathcal{E}^{\dagger} \log \mathcal{E}(\sigma)$ and $Z^{(t+1)}=$ $\left.\operatorname{Tr}\left[\exp \left(\log \rho^{(t)}+\frac{1}{\gamma} \mathcal{F}\left(\rho^{(t)}\right)\right)\right)\right]$ normalizes the state. To calculate the complexity of applying the update rule, we use that

- the quantum channel $\mathcal{E}$, its adjoint $\mathcal{E}^{\dagger}$, the complementary channel $\mathcal{E}_{c}$ and its adjoint $\mathcal{E}_{c}^{\dagger}$ are all given as lookup tables whose $(i, j)$-th entry is given by $\mathcal{E}(|i\rangle\langle j|)$. We assume constant time access to the entries of the table. Hence, the application of the quantum channel $\mathcal{E}_{A \rightarrow B}$ and of its adjoint have time complexity $\mathcal{O}\left(|A|^{2}|B|^{2}\right)$ whereas the 
TABLE I

RESULTS FOR CLASSICAL, CLASSICAL-QUANTUM AND QUANTUM CHANNELS USING NON-COMMUTATIVE BLAHUT-ARIMOTO ALGORITHMS.

\begin{tabular}{cccc}
\hline Channels & Quantity & Optimization problem & Time complexity \\
\hline$X \rightarrow Y$ & Mutual information $I(\mathcal{E})$ & $\max _{\lambda} \sum_{i} \lambda_{i} \operatorname{Tr}\left[\tau_{\mathcal{E}, i}\left\{\log \tau_{\mathcal{E}, i}-\log \mathcal{E}\left(\rho_{\lambda}\right)\right\}\right]$ & $\mathcal{O}\left(\frac{|X||Y| \log |X|}{\varepsilon}\right)$ \\
$X \rightarrow B$ & Holevo quantity $\chi(\mathcal{E})$ & $\max _{\lambda} \sum_{i} \lambda_{i} \operatorname{Tr}\left[\tau_{\mathcal{E}, i}\left\{\log \tau_{\mathcal{E}, i}-\log \mathcal{E}\left(\rho_{\lambda}\right)\right\}\right]$ & $\mathcal{O}\left(\frac{\left(|B|^{3}+|B|^{2}|X|\right) \log |X|}{\varepsilon}+|X||B|^{3}\right)$ \\
$A \rightarrow B$ & Thermodynamic capacity $T(\mathcal{E})$ & $\max _{\rho} S(\rho)-S(\mathcal{E}(\rho))$ & $\mathcal{O}\left(\frac{\left(|A|^{3}+|A|^{2}|B|^{2}+|B|^{3}\right) \log |A|}{\varepsilon}\right)$ \\
$A \rightarrow B$ & Coherent information $I_{\operatorname{coh}}(\mathcal{E})$ & $\max _{\rho} S(\mathcal{E}(\rho))-S\left(\mathcal{E}_{c}(\rho)\right)$ & $\mathcal{O}\left(\frac{\left(|A|^{3}+|B|^{3}+K^{3}+|A|^{2}\left(|B|^{2}+K^{2}\right)\right) \log |A|}{\varepsilon}\right)$ \\
$A \rightarrow B$ & Quantum mutual information $I(\mathcal{E})$ & $\max _{\rho} S(\rho)+S(\mathcal{E}(\rho))-S\left(\mathcal{E}_{c}(\rho)\right)$ & $\mathcal{O}\left(\frac{\left(|A|^{3}+|B|^{3}+K^{3}+|A|^{2}\left(|B|^{2}+K^{2}\right)\right) \log |A|}{\varepsilon}\right)$ \\
\hline
\end{tabular}

application of the channel $\left(\mathcal{E}_{c}\right)_{A \rightarrow E}$ and of its adjoint have time complexity $\mathcal{O}\left(|A|^{2}|E|^{2}\right)$ and

- the complexity of calculating the matrix logarithm and exponential of a density operator $\rho_{A}$ is $\mathcal{O}\left(|A|^{3}\right)$.

An iteration step of the form given in (26) is then found to have complexity $\mathcal{O}\left(|A|^{3}+|B|^{3}+|E|^{3}+|A|^{2}\left(|B|^{2}+|E|^{2}\right)\right)$. By Stinesprings theorem, the dimension of the environment can be bounded by $|E| \leq K$, where $K \leq|A||B|$ denotes the Kraus rank of $\mathcal{E}$, i.e., the minimal number of Kraus operators required to represent $\mathcal{E}$. Hence, the complexity of one iteration step is $\mathcal{O}\left(|A|^{3}+|B|^{3}+K^{3}+|A|^{2}\left(|B|^{2}+K^{2}\right)\right)$. The number of required iterations to get an $\varepsilon$ approximation to the capacity is of order $\mathcal{O}(\log |A| / \varepsilon)$ and hence we conclude that the time complexity of Algorithm 2 is given by

$$
\mathcal{O}\left(\frac{\left(|A|^{3}+|B|^{3}+K^{3}+|A|^{2}\left(|B|^{2}+K^{2}\right)\right) \log |A|}{\varepsilon}\right) .
$$

\section{AdDitional SETTINGS}

Similar to the setting in Section IV, we may also estimate other entropic optimization problems by bringing them into the standard form (2) in the Definition 3.1 of Blahut-Arimoto algorithms. For all the cases discussed in the following, the form of $\mathcal{F}(\sigma)$ satisfies (7), and this allows us to prove convergence using Theorem 3.3. Table I has more details about the time complexity to approximate an additive error of $\varepsilon$. Details about accelerating the algorithm, proving exponential convergence, and numerical results for these quantities can be found in the full version [28].

\section{A. Quantum mutual information}

The entanglement-assisted classical capacity represents the maximum rate at which Alice can reliably send a classical message to Bob through a quantum channel while using shared entanglement. It is given by the mutual information $I(\mathcal{E})$ of quantum channels $\mathcal{E}_{A \rightarrow B}$ [31], defined as

$$
I(\mathcal{E})=\max _{\rho} S(\rho)+S(\mathcal{E}(\rho))-S\left(\mathcal{E}_{c}(\rho)\right) .
$$

We may bring this into standard form by choosing

$$
\mathcal{F}(\sigma)=\mathcal{E}_{c}^{\dagger} \log \mathcal{E}_{c}(\sigma)-\log (\sigma)-\mathcal{E}^{\dagger} \log \mathcal{E}(\sigma)
$$

\section{B. Thermodynamic capacity}

The thermodynamic capacity quantifies the informationtheoretic power of quantum channels in the presence of physical restrictions imposed by thermodynamics [32]. For a quantum channel $\mathcal{E}_{A \rightarrow B}$, it can be written as (a more general form is provided in the full version [28])

$$
T(\mathcal{E})=\max _{\rho} S(\rho)-S(\mathcal{E}(\rho))
$$

We can bring this into standard form by choosing

$$
\mathcal{F}(\sigma)=\mathcal{E}^{\dagger}(\log \mathcal{E}(\sigma))-\log \sigma
$$

\section{Holevo quantity}

Classical-quantum channels are defined as $\mathcal{E}_{X \rightarrow B}: \rho_{X} \mapsto$ $\sum_{i}\left\langle i\left|\rho_{X}\right| i\right\rangle\left(\tau_{i}\right)_{B}$. Classical channels are included in this formalism by considering the case where all $\left(\tau_{i}\right)_{B}$ are diagonal in the basis $\{|i\rangle\}$. The Holevo quantity that quantifies the classical channel capacity [1], [2] is defined as

$$
\chi(\mathcal{E})=\max _{\lambda} \sum_{i} \lambda_{i} \operatorname{Tr}\left[\tau_{\mathcal{E}, i}\left\{\log \tau_{\mathcal{E}, i}-\log \mathcal{E}\left(\rho_{\lambda}\right)\right\}\right]
$$

with $\tau_{\mathcal{E}, i}=\mathcal{E}(|i\rangle\langle i|)$. We can bring this into standard form with

$$
\mathcal{F}(\sigma)=\sum_{i}|i\rangle\langle i| \operatorname{Tr}[\mathcal{E}(|i\rangle\langle i|)(\log \mathcal{E}(|i\rangle\langle i|)-\log \mathcal{E}(\sigma))] .
$$

This recovers the work [3], [27].

\section{CONCLUSION}

We gave an analytical and numerical analysis of alternating optimization algorithms of Blahut-Arimoto type for computing various channel capacities in quantum information theory. We note that our algorithms are of zeroth-order and do not need to take into account matrix valued derivatives. Thus, they are rather straightforward and computationally inexpensive to implement - as demonstrated in our numerical example. It remains open if alternating optimization algorithms, in particular of Blahut-Arimoto type, can also be given for other optimization problems in terms of quantum entropy. 


\section{REFERENCES}

[1] A. S. Holevo, "The capacity of the quantum channel with general signal states," IEEE Transactions on Information Theory, vol. 44, no. 1, pp. 269-273, 1998.

[2] B. Schumacher and M. D. Westmoreland, "Sending classical information via noisy quantum channels," Physical Review A, vol. 56, no. 1, pp. 131$138,1997$.

[3] H. Nagaoka, "Algorithms of Arimoto-Blahut type for computing quantum channel capacity," in IEEE International Symposium on Information Theory, 1998, p. 354.

[4] S. Osawa and H. Nagaoka, "Numerical experiments on the capacity of quantum channel with entangled input states," IEICE Transactions Fundamentals, vol. E84-A, no. 10, pp. 2583-2590, 2001.

[5] P. W. Shor, "Capacities of quantum channels and how to find them," Mathematical Programming, vol. 97, no. 1, pp. 311-335, 2003.

[6] M. Hayashi, H. Imai, K. Matsumoto, M. B. Ruskai, and T. Shimono, "Qubit channels which require four inputs to achieve capacity: Implications for additivity conjectures," Quantum Information and Computation, vol. 5, no. 1, pp. 13-31, 2005.

[7] K. Kato, M. Oto, H. Imai, and K. Imai, "Computational geometry analysis of quantum state space and its applications," in Generalized Vorono Diagram: A Geometry-Based Approach to Computational Intelligence. Springer, 2009, pp. 67-108.

8] T. Tanuma, H. Imai, and S. Moriyama, "Revisiting hyperbolic vorono diagrams in two and higher dimensions from theoretical, applied and generalized viewpoints," in Transactions on Computational Science XIV. Springer, 2011, pp. 1-30.

[9] D. Sutter, T. Sutter, P. Mohajerin Esfahani, and R. Renner, "Efficient approximation of quantum channel capacities," IEEE Transactions on Information Theory, vol. 62, no. 1, pp. 578-598, 2016

[10] H. Fawzi and O. Fawzi, "Efficient optimization of the quantum relative entropy," Journal of Physics A: Mathematical and Theoretical, vol. 51, no. 15 , p. 154003,2018

[11] H. Fawzi, J. Saunderson, and P. A. Parrilo, "Semidefinite approximations of the matrix logarithm," Foundations of Computational Mathematics, vol. 19, no. 2, pp. 259-296, 2019.

[12] Y. Zinchenko, S. Friedland, and G. Gour, "Numerical estimation of the relative entropy of entanglement," Physical Review A, vol. 82, no. 5, p. 052336, 2010.

[13] M. W. Girard, G. Gour, and S. Friedland, "On convex optimization problems in quantum information theory," Journal of Physics A: Mathematical and Theoretical, vol. 47, no. 50, p. 505302, 2014.

[14] R. Blahut, "Computation of channel capacity and rate-distortion functions," IEEE Transactions on Information Theory, vol. 18, no. 4, pp 460-473, 1972.

[15] S. Arimoto, "An algorithm for computing the capacity of arbitrary discrete memoryless channels," IEEE Transactions on Information Theory, vol. 18, no. 1, pp. 14-20, 1972.

[16] N. Tishby, F. C. Pereira, and W. Bialek, "The information bottleneck method," in Annual Allerton Conference on Communication, Control and Computing, 1999, pp. 368-377.

[17] P. O. Vontobel, "A generalized Blahut-Arimoto algorithm," in IEEE International Symposium on Information Theory, 2003, p. 53.

[18] M. Rezaeian and A. Grant, "Computation of total capacity for discrete memoryless multiple-access channels," IEEE Transactions on Information Theory, vol. 50, no. 11, pp. 2779-2784, 2004

[19] F. Dupuis, W. Yu, and F. M. J. Willems, "Blahut-Arimoto algorithms for computing channel capacity and rate-distortion with side information," in International Symposium on Information Theory, 2004, p. 179.

[20] I. Naiss and H. H. Permuter, "Extension of the blahut-arimoto algorithm for maximizing directed information," IEEE Transactions on Information Theory, vol. 59, no. 1, pp. 204-222, 2013.

[21] C.-I. Chang and L. D. Davisson, "On calculating the capacity of an infinite-input finite (infinite)-output channel," IEEE Transactions on Information Theory, vol. 34, no. 5, pp. 1004-1010, 1988.

[22] J. Huang and S. P. Meyn, "Characterization and computation of optimal distributions for channel coding," IEEE Transactions on Information Theory, vol. 51, no. 7, pp. 2336-2351, 2005.

[23] J. Sayir, "Iterating the Arimoto-Blahut algorithm for faster convergence," in IEEE International Symposium on Information Theory, 2000, p. 235

[24] G. Matz and P. Duhamel, "Information geometric formulation and interpretation of accelerated Blahut-Arimoto-type algorithms," in Information Theory Workshop, 2004, pp. 66-70.
[25] Z. Naja, F. Alberge, and P. Duhamel, "Geometrical interpretation and improvements of the Blahut-Arimoto's algorithm," in IEEE International Conference on Acoustics, Speech and Signal Processing, 2009, pp. 2505-2508.

[26] Y. Yu, "Squeezing the Arimoto-Blahut algorithm for faster convergence," IEEE Transactions on Information Theory, vol. 56, no. 7, pp. 3149-3157, 2010

[27] H. Li and N. Cai, "A Blahut-Arimoto type algorithm for computing classical-quantum channel capacity," in IEEE International Symposium on Information Theory, 2019, pp. 255-259.

[28] N. Ramakrishnan, R. Iten, V. B. Scholz, and M. Berta, "Non-commutative Blahut-Arimoto algorithms," arXiv preprint arXiv:1905.01286, 2019.

[29] S. Watanabe, "Private and quantum capacities of more capable and less noisy quantum channels," Physical Review A, vol. 85, no. 1, p. 012326 , 2012.

[30] I. Devetak, "The private classical capacity and quantum capacity of a quantum channel," IEEE Transactions on Information Theory, vol. 51, no. 1, pp. 44-55, 2005.

[31] C. H. Bennett, P. W. Shor, J. A. Smolin, and A. V. Thapliyal, "Entanglement-assisted capacity of a quantum channel and the reverse Shannon theorem," IEEE Transactions on Information Theory, vol. 48, no. 10, pp. 2637-2655, 2002.

[32] P. Faist, M. Berta, and F. Brandão, "Thermodynamic capacity of quantum processes," Physical Review Letters, vol. 122, no. 20, p. 200601, 2019. 